\title{
SYNTHESES, ANTIMICROBIAL ACTIVITY AND MASS SPECTRAI INVESTIGATION OF SOME NEW THIOHYDANTOIN AND THIAZOLE DERIVATIVES.
}

\author{
H. Abd El Hady ${ }^{* 1}$ \\ *Chemistry Department, Faculty of Science (For Girls), Al -Azhar University Nasr City, \\ Egypt. \\ ${ }^{1}$ Current address : Umm- al qura University Faculty of applied Science ( For Girls) \\ ,Chemistry Department .
}

\begin{abstract}
5-(p-Tolyl)-2-[(p-tolylethylidene)hydrazino] thiazole (3) and 3-[(ptolylethylidene )amino]-2-thiohydantoin (6) have been prepared via cyclization of $\mathrm{p}$ methyl acetophenone thiosemicarbazone (2) with p-methylphenacyl bromide and ethyl chloroacetate in presence of fused sodium acetate. Acetylation of 2,3 and 6 with acetic anhydride afforded the corresponding diacetyl derivative(5) and mono acetyl derivatives (4 and 7). Reaction of 2-thiohydantoin (6) with thiophene-2-carboxaldhyde and chloroacetic acid gives 5-(thiophen-2-ylidene)-3-[(p-tolylethylidene)amino] -2-thiohydantoin (8) and 3[(p- tolylethylidene )amino] -2-thiohydantoin-5-yl acetic acid (10). The mass spectral fragmentation patterns of some prepared thiazole and 2-thiohydantoin derivatives have been investigated in order to elucidate the structure of the synthesized compounds. The prepared compounds also exhibited antimicrobial activity.
\end{abstract}

Graphical abstract<smiles>CCOC(=O)COC(N)=S</smiles><smiles>[R]N=C(C)c1ccc(C)cc1</smiles><smiles>Cc1cccc(-c2cnc(NN)s2)c1</smiles>

Key words: Thiohydantoin , thiazole , thiosemicarbazone , anti-bacterial activity , antifungal activity, minimum inhibition concentration .

\section{INTRODUCTION}

The chemistry and properties of thiohydantoins and their derivatives have been investigated for more than 145 years. The hydantoin moiety represents an important pharmacophore, which is present in various biologically active compounds. The 1aminohydantoin (Wilson and Portlock, 1998; Behai, 2003) is an antimicrobial drug for the treatment of urinary tract infections, while its analog dantrolene (Wilson and Portlock, 
1998; Behai, 2003) represents a well known skeletal muscle relaxant. Another 1aminohydantoin, azmilide, is a promising drug candidate for the treatment of cardiac arrhythmia. Phenylhydantoin, (Brouilette, et al., 1994) 5,5-diphenylhydantoin is an anticonvulsant used for the treatment of epilepsy. This paper now reports the synthesis of thiazole and 2-thiohydantoin derivatives by using p-methyl acetophenone thiosemicarbazone (2) as a key starting material. The electron impact (EI) ionization mass spectral fragmentation patterns of some synthesized compounds are described.

\section{RESULTS AND DISCUSSION}

The reaction of 4-methylacetophenone (1) with thiosemicarbazide in methanol under reflux gave the corresponding 4-methylacetophenone thiosemicarbazone (2). Treatment (Mohamed, et al., 2006a; Mohamed, et al., 2006b) of compound 2 with 4methylpheynacyl bromide and ethyl chloroacetate in presence of fused sodium acetate in ethanol under reflux yielded the corresponding 5-(p-tolyl)-2- [(p-tolylethylidene)hydrazino] thiazole (3) and 3-[(p-tolylethylidene )amino]-2-thiohydantoin (6). Acetylation of thiosemicarbazone (2), thiazole (3) and 2-thiohydantoin (6) with acetic anhydride under reflux led to the formation of 4-methylacetophenone - 2,4-diacetyl thiosemicarbazone (5), 2-[(p-tolylethylidene)acetyl hydrazino]-thiazole (4) and 1-acetyl-3-[(p-tolylethylidene) amino]-2-thiohydantoin (7), respectively.

Condensation (Kiec and Szyman, 2002) of 3-[(p-tolylethylidene)amino]-2thiohydantoin (6) with thiophene-2- carboxaldehyde in presence of piperidine under reflux led to the formation of 5-thiophen-2-ylidene-3-[(p-tolylethylidene)amino]-2-thiohydantoin (8,Scheme1). Acylation of compound 8 with acetic anhydride afforded the corresponding 1-acetyl-3- [(p-tolylethylidene)amino]-5-thiophen-2-ylidene-2-thiohydantoin (9). The structure of 9 was also established via reaction of compound 7 with thiophene-2carboxaldehyde in presence of piperidine.

Heating (Hasanen, 2007) of 2-thiohydantoin (6) with chloroacetic acid in xyline in the presence of sodium metal under reflux gave the corresponding 3-[(ptolylethylidene)amino]-2-thiohydantoin-5-yl acetic acid (10,Scheme1).

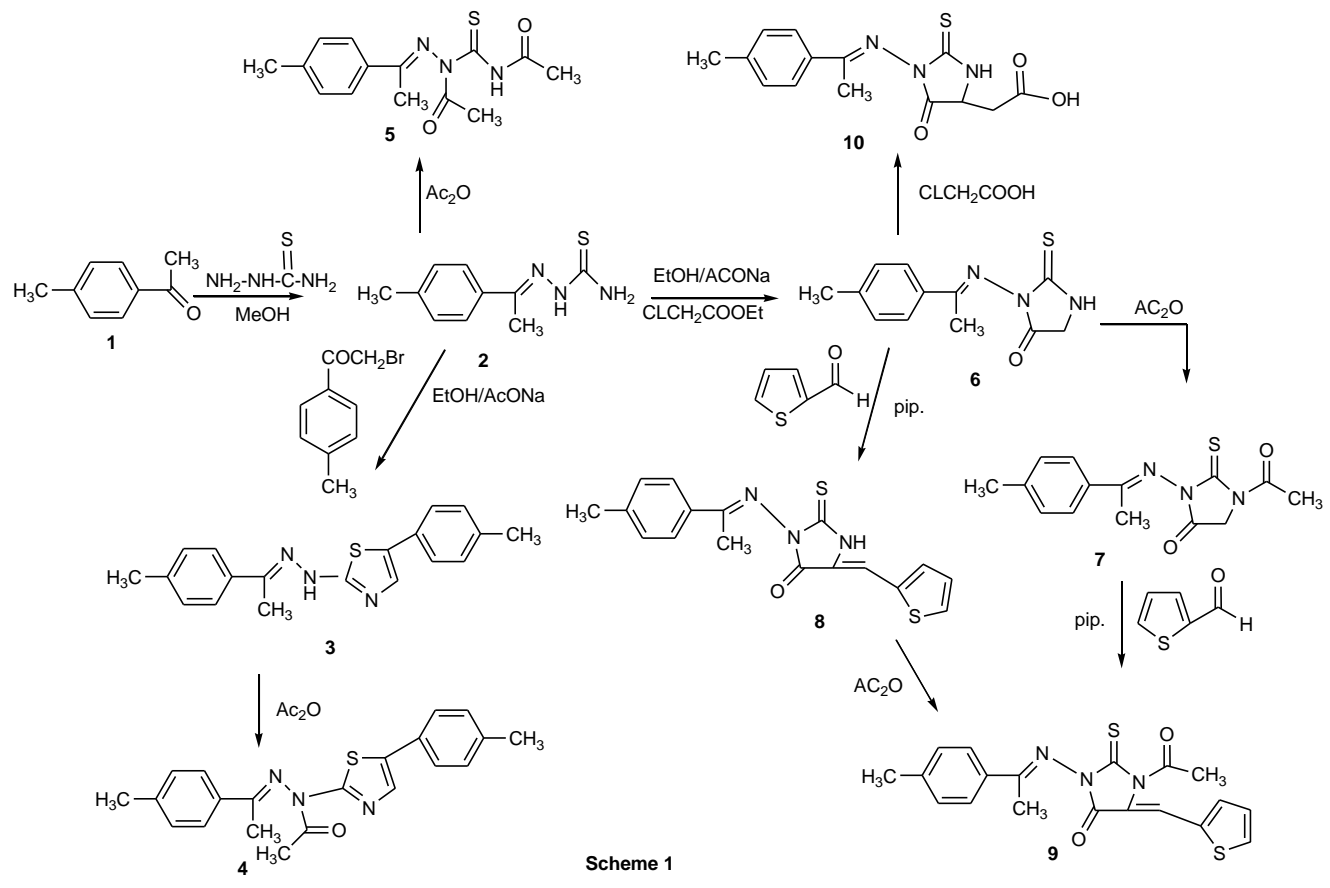




\section{Mass spectrometry}

The mass spectral decomposition modes (El-Deen and Ibrahim, 2004) of various thiazoles and 2-thiohydantoin derivatives containing p-tolylethylidene amino substituents have been investigated and fragmentation pathways have been suggested.

\section{Compounds 3 and 4}

The mass spectra of the synthesized compounds 3 and 4 (Figure1) showed intense molecular ion peaks at $\mathrm{m} / \mathrm{z} 321, \mathrm{~m} / \mathrm{z} 363$, consistent with the molecular formula $\mathrm{C}_{19} \mathrm{H}_{19} \mathrm{~N}_{3} \mathrm{~S}$ and $\mathrm{C}_{21} \mathrm{H}_{21} \mathrm{~N}_{3} \mathrm{OS}$, respectively. From the study of the mass spectra of compounds $\mathbf{3}$ and $\mathbf{4}$ it was found that the molecular ion of all these compounds fragmented further, along two different pathways, as illustrated by (Scheme 2). The molecular ion of $\mathrm{m} / \mathrm{z} 321$ for compound 3 fragmented via pathway $\mathbf{A}$ to gave the fragment of m/z 132 by losing 5-(ptolyl)-2-amino-thiazole radical cation. The fragment of $\mathrm{m} / \mathrm{z} \mathrm{132}$, fragmented to give the fragment of $\mathrm{m} / \mathrm{z} 117$ by losing methyl group. This fragment of $\mathrm{m} / \mathrm{z} 117$ fragmented more to

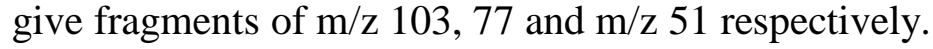

Accordingly, the same molecular ion of $\mathrm{m} / \mathrm{z} 321$ fragmented via the pathway $\mathbf{B}$ to give the ion of $\mathrm{m} / \mathrm{z} 189$, corresponding to 5-(p-tolyl)-2-aminothiazole, which lost nitrogen atom to give the ion of $\mathrm{m} / \mathrm{z} 175$. The ion of $\mathrm{m} / \mathrm{z} 175$ was broken to give an ion of $\mathrm{m} / \mathrm{z} 148$ by losing hydrogen cyanide. The ion at $\mathrm{m} / \mathrm{z} 148$ underwent loss of sulfur atom and acetylene cation $\left(\mathrm{C}_{2} \mathrm{H}\right)$ to give peaks at $\mathrm{m} / \mathrm{z} 116$ and $\mathrm{m} / \mathrm{z} 91$ respectively.

From the mass spectrum of compound 4, it was concluded that the molecular ion was at $\mathrm{m} / \mathrm{z} 363$. The ion of $\mathrm{m} / \mathrm{z} 363$ under went fragmentation to produce a stable ion at $\mathrm{m} / \mathrm{z}$ 321 by losing ketene molecule $\left(\mathrm{CH}_{2} \mathrm{CO}\right)$, corresponding to the molecular ion of compound 3. The stable fragment of $\mathrm{m} / \mathrm{z} 321$ further broken via pathway similar to compound 3 (Scheme 2).

The mass spectrum of compound 5 (Figure 2) shows the molecular ion peak at $\mathrm{m} / \mathrm{z}$ 291 , corresponding to the molecular formula $\mathrm{C}_{14} \mathrm{H}_{17} \mathrm{~N}_{3} \mathrm{O}_{2} \mathrm{~S}$. The molecular ion of $\mathrm{m} / \mathrm{z} 291$ for compound $\mathbf{5}$ fragmented further a long two pathways as illustrated in (Scheme 3) .

\section{Compounds 6 and 7}

The mass spectrum of compound $\mathbf{6}$ showed an intense molecular ion peak at $\mathrm{m} / \mathrm{z}$ 247 , corresponding to the molecular formula $\mathrm{C}_{12} \mathrm{H}_{13} \mathrm{~N}_{3} \mathrm{OS}$. The molecular ion of compound 6 (Figure 3) underwent fragmentation via pathway A to produce a peak at $\mathrm{m} / \mathrm{z} 233$ by losing nitrogen atom. The loss of acetyl group from the ion with $\mathrm{m} / \mathrm{z} 233$ resulted in ion at $\mathrm{m} / \mathrm{z}$ 190. The ion of $\mathrm{m} / \mathrm{z} 190$ broken to give an ion at $\mathrm{m} / \mathrm{z} 132$ which lost isothiocynate group (NCS). It further underwent loss of two methyl groups and hydrogen cyanide to give peaks at $\mathrm{m} / \mathrm{z} 117,102$ and 75, respectively.

Also, the molecular ion of compound 6 at m/z 247 underwent loss of $\mathrm{NH}$ group via pathway $\mathbf{B}$ to give a stable ion at $\mathrm{m} / \mathrm{z} 232$. The loss of carbon monoxide from the ion at $\mathrm{m} / \mathrm{z}$ 232 resulted in an ion at m/z 204. The ion of m/z 204 broken to give an ion at $\mathrm{m} / \mathrm{z} 159$ by losing thioformyl group (CHS). The ion of m/z 159 fragmented to give an ion of $\mathrm{m} / \mathrm{z} 133$ by losing a cyano group $(\mathrm{CN})$. It further underwent loss of methyl group, hydrogen cyanide and acetylene molecules to give peaks at $\mathrm{m} / \mathrm{z} 118,91$ and $\mathrm{m} / \mathrm{z} 65$, respectively.

The molecular ion of compound 7 (m/z 289) had fragmented to give the fragment of $\mathrm{m} / \mathrm{z} 247$, corresponding to the molecular ion of compound $\mathbf{6}$ by losing ketene molecule $\left(\mathrm{CH}_{2} \mathrm{CO}\right)$. The fragment of $\mathrm{m} / \mathrm{z} 247$ was broken via pathway in the same fragmented processes which was observed for compound 6 (Scheme 4). 


\section{Compounds 8 and 9}

The mass spectra of compounds $\mathbf{8}$ and 9are fully consistent with the assigned structures, in most cases, intense molecular ion peak was observed. Thus compound 8 showed an intense molecular ion peak at $\mathrm{m} / \mathrm{z} 341$ (Figure 5) corresponding to the molecular formula $\mathrm{C}_{17} \mathrm{H}_{15} \mathrm{~N}_{3} \mathrm{OS}_{2}$. The molecular ion peak of compound $\mathbf{9}$ was observed at $\mathrm{m} / \mathrm{z} 383$ (Figure 6) corresponding to the molecular formula $\mathrm{C}_{19} \mathrm{H}_{17} \mathrm{~N}_{3} \mathrm{O}_{2} \mathrm{~S}_{2}$. The main fragmentation pathways of compounds 8 and $\mathbf{9}$ are summarized in (Scheme )5 .

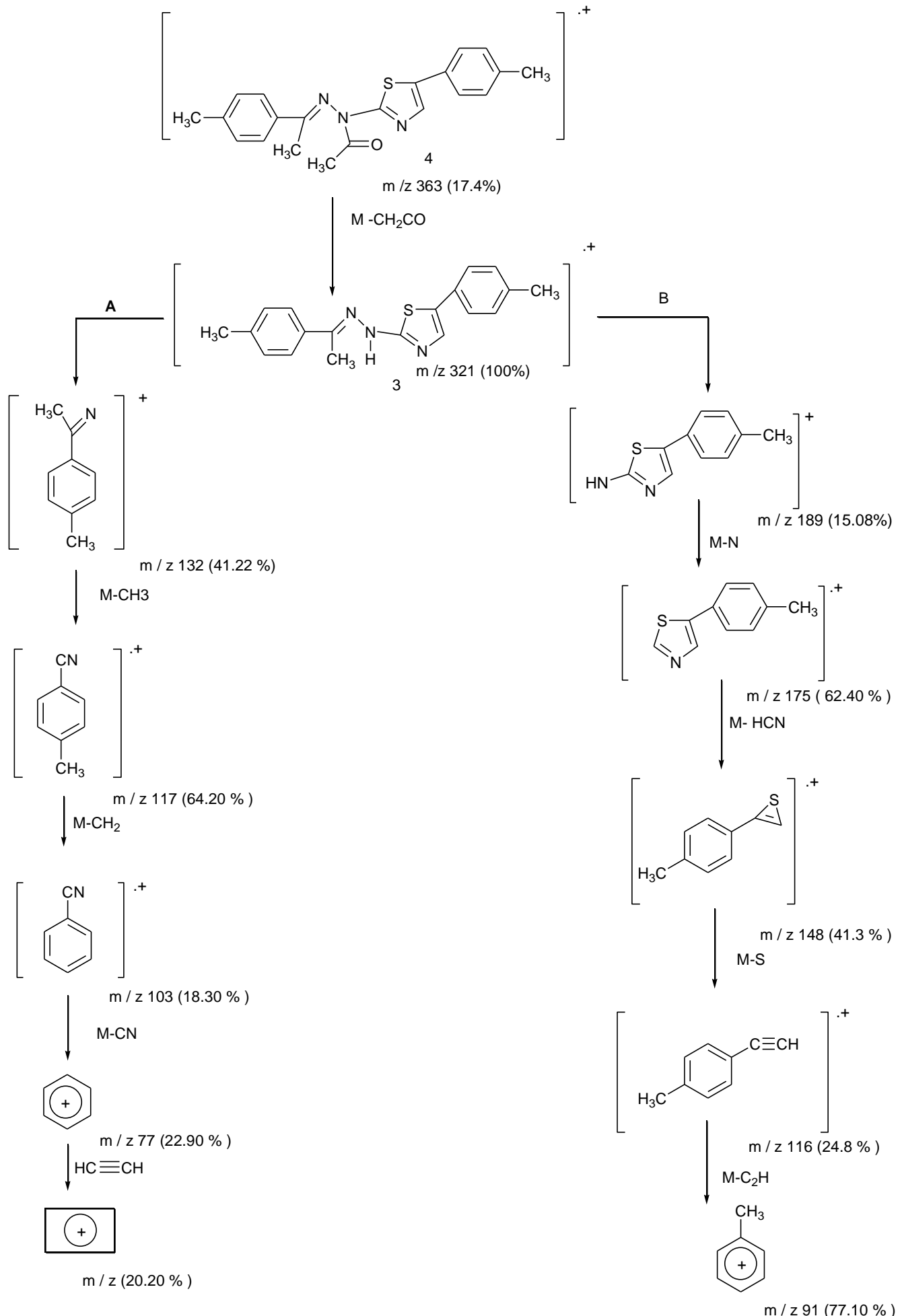



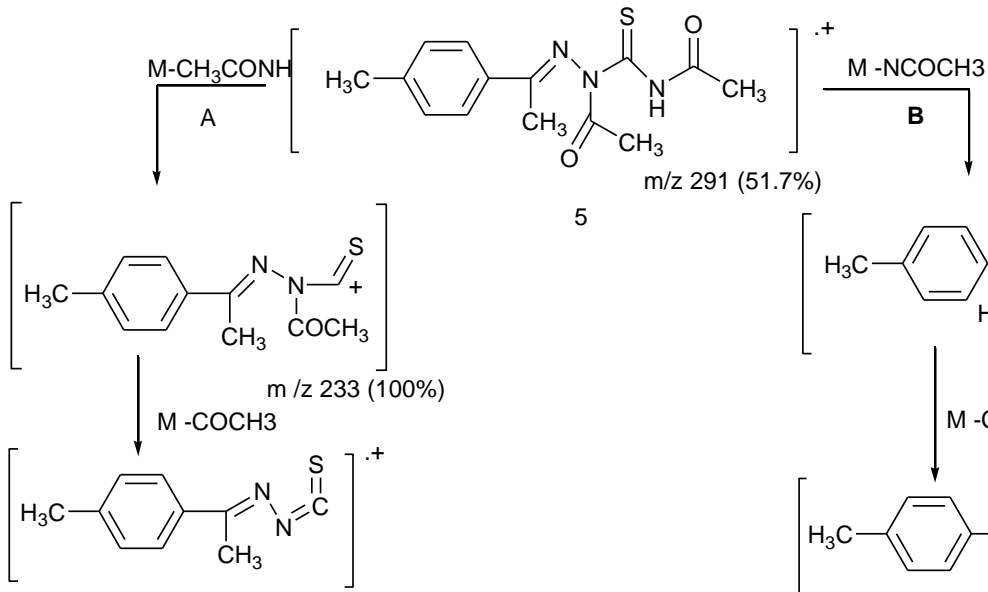

5

$\mathrm{m} / \mathrm{z} 190(72.4 \%)$

M - NCS

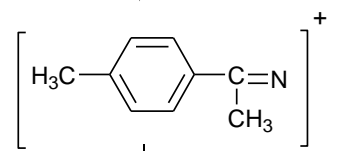

$\int_{\mathrm{M}-\mathrm{CH} 3}^{\mathrm{m} / \mathrm{z} 132 \text { (75.9\%) }}$

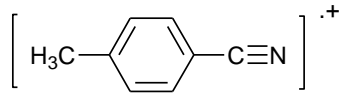

$\mid \begin{aligned} & \mathrm{m} / \mathrm{z} 117 \text { (79.3\%) } \\ & \mathrm{M}-\mathrm{CH}_{2}\end{aligned}$
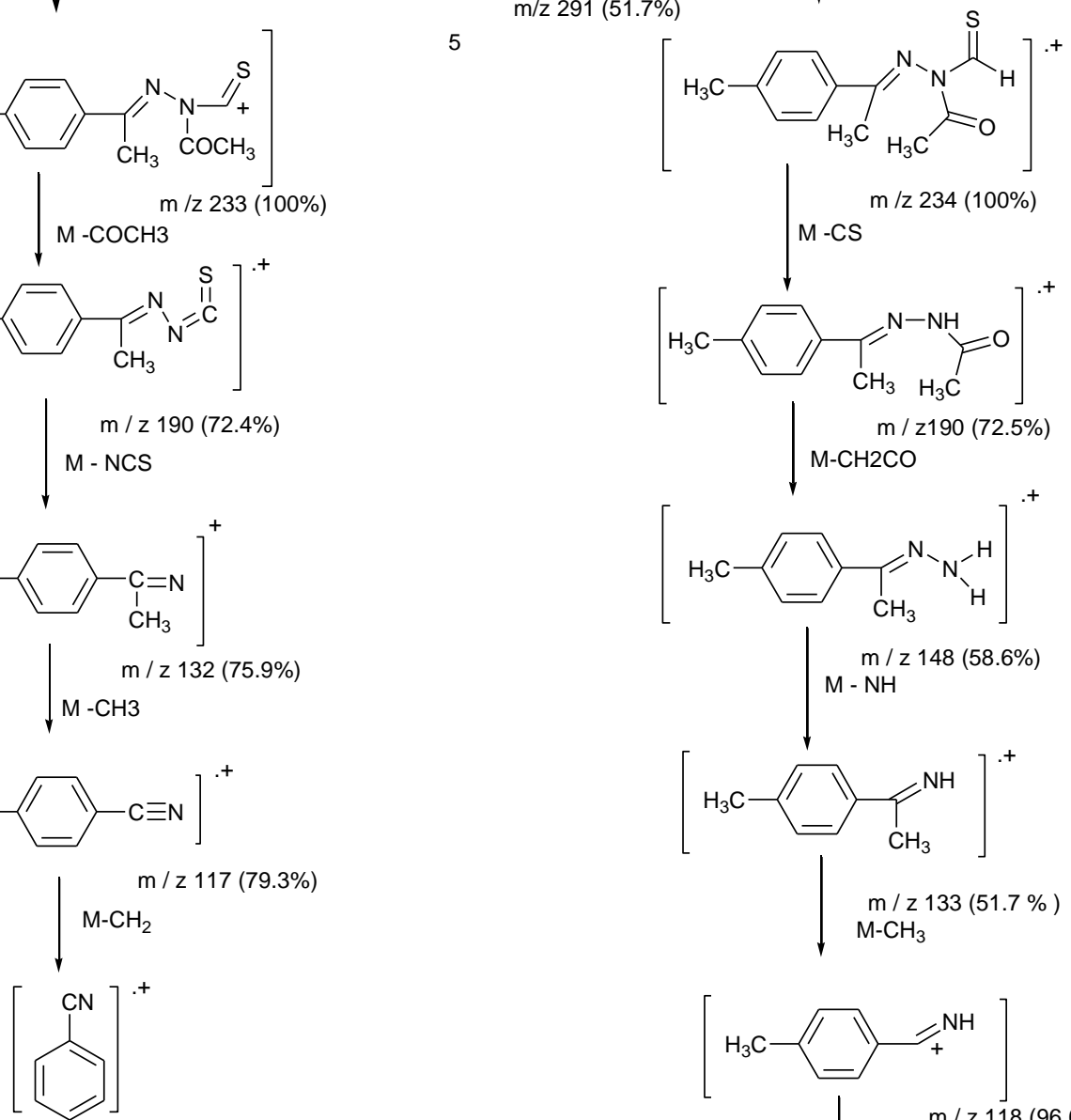

$\mathrm{m} / \mathrm{z} 103(34.5 \%)$

$\mathrm{M}-\mathrm{CN}$

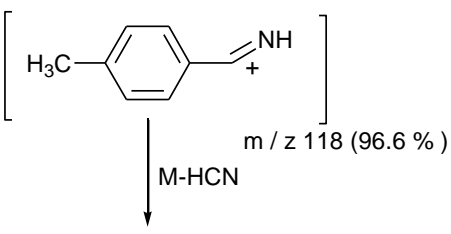

(

$\mathrm{m} / \mathrm{z} 77(24.5 \%)$

$\mathrm{m} / \mathrm{z} 91(51.7 \%)$

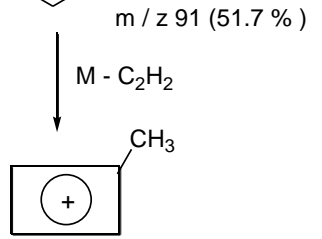

$\mathrm{m} / \mathrm{z} 65(65.5 \%)$

Scheme 3:Main fragmentation pathways of compound 5 

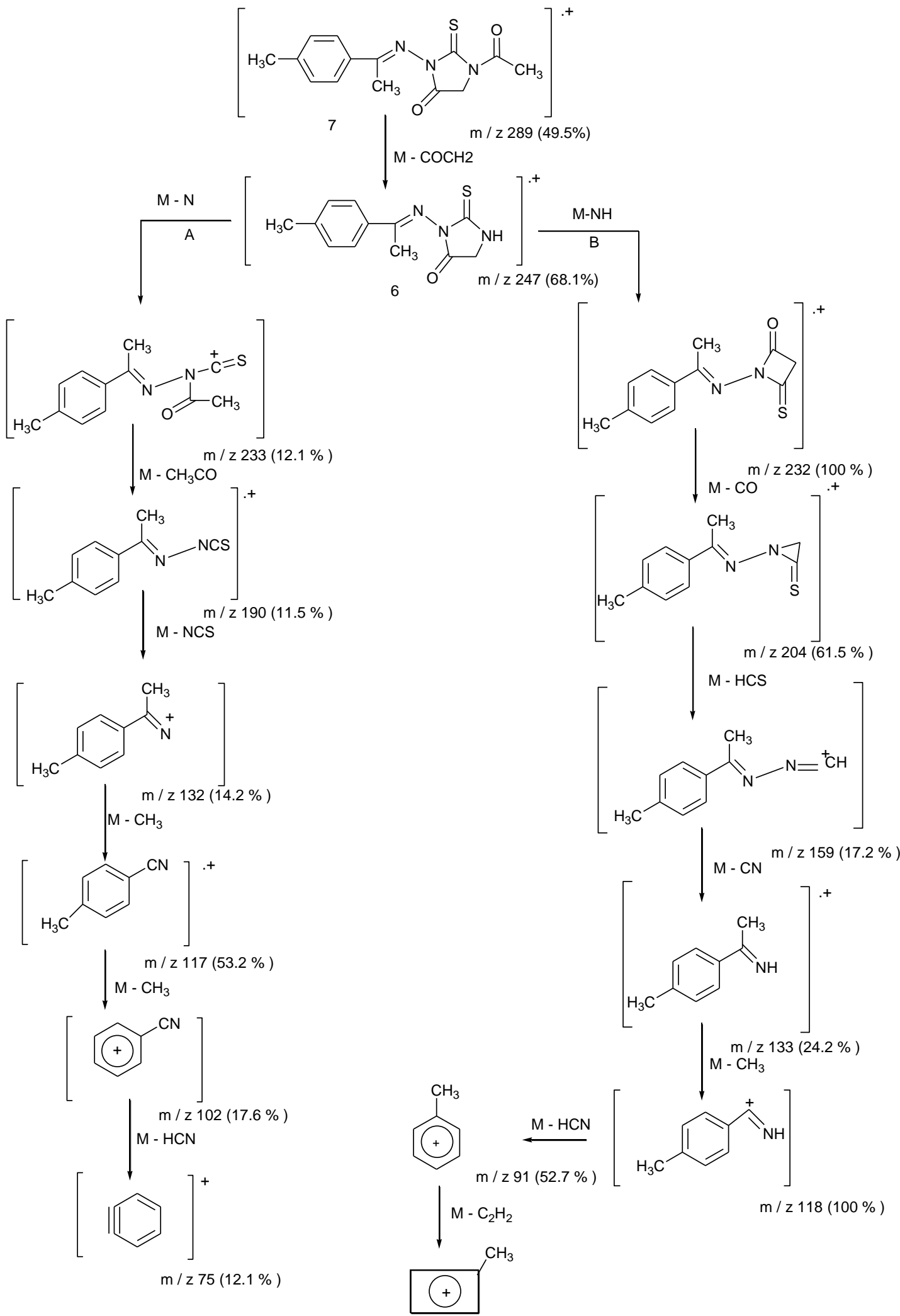

$\mathrm{m} / \mathrm{z} 65(47.3 \%)$ 


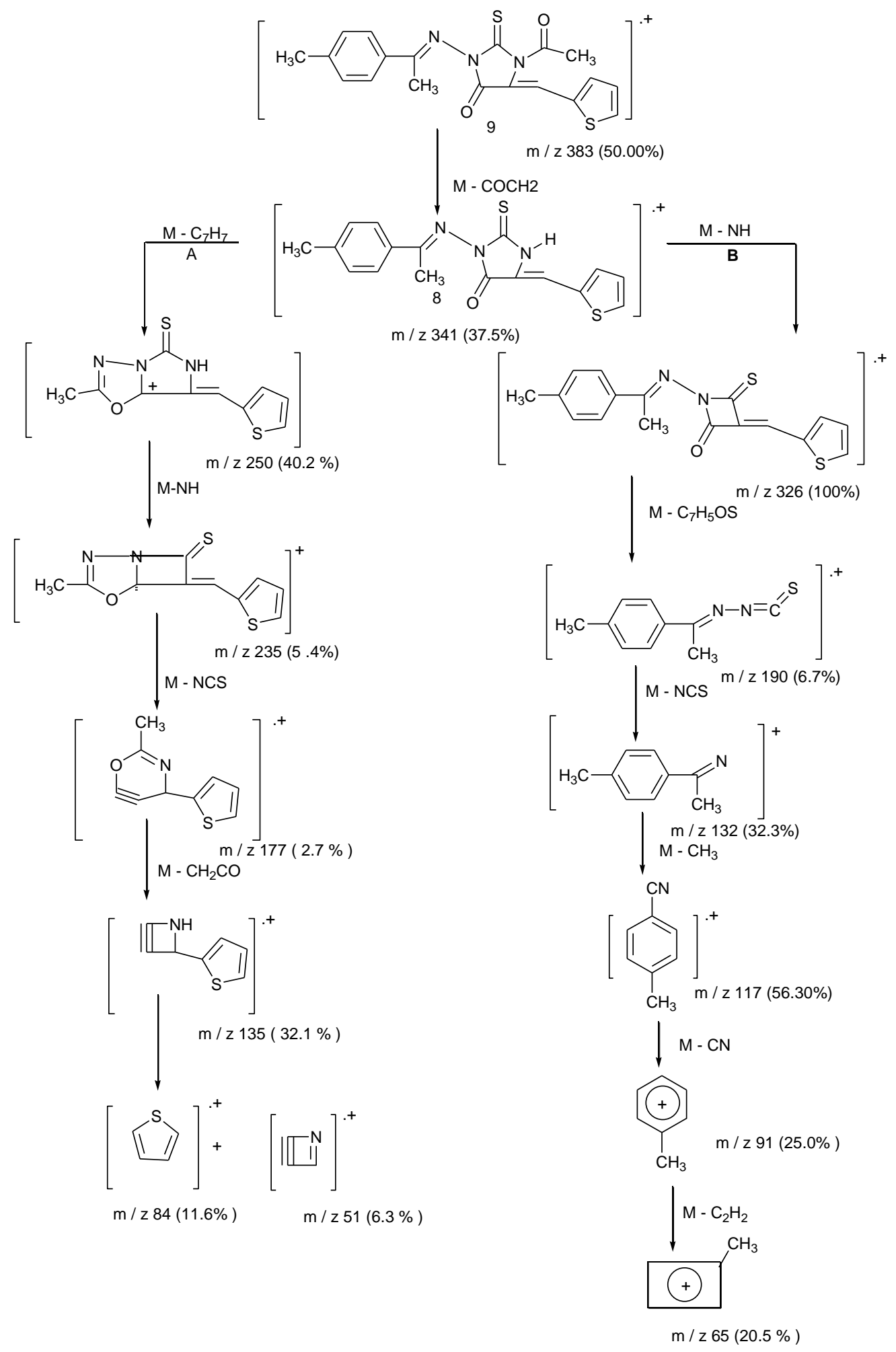

Scheme 5: Main fragmentation pathways of compounds 8 and 9 


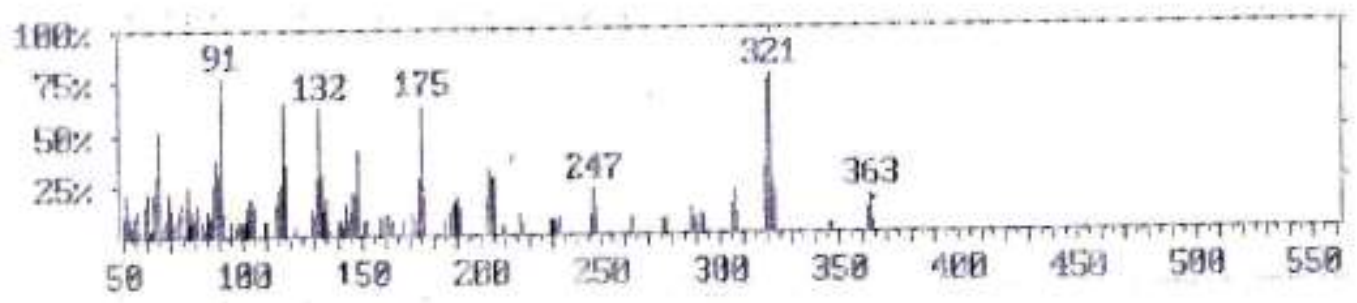

Fig. (1) Mass spectrum (70ev) of compound 4

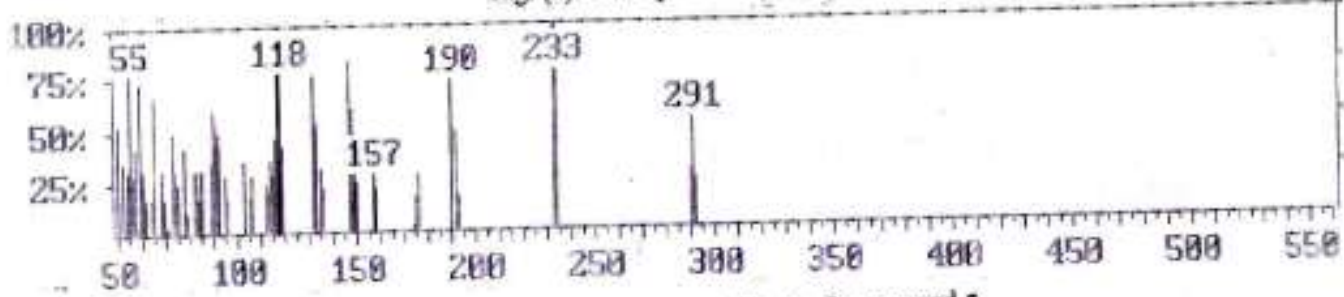

Fig. (2) Mass spectrum (70ev) of compound 5

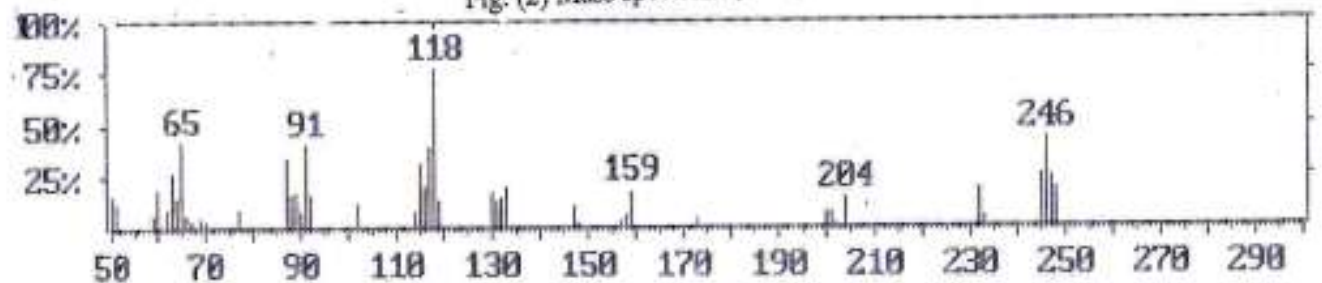

Fig. (3) Mass spectrum (70ev) of compound 6
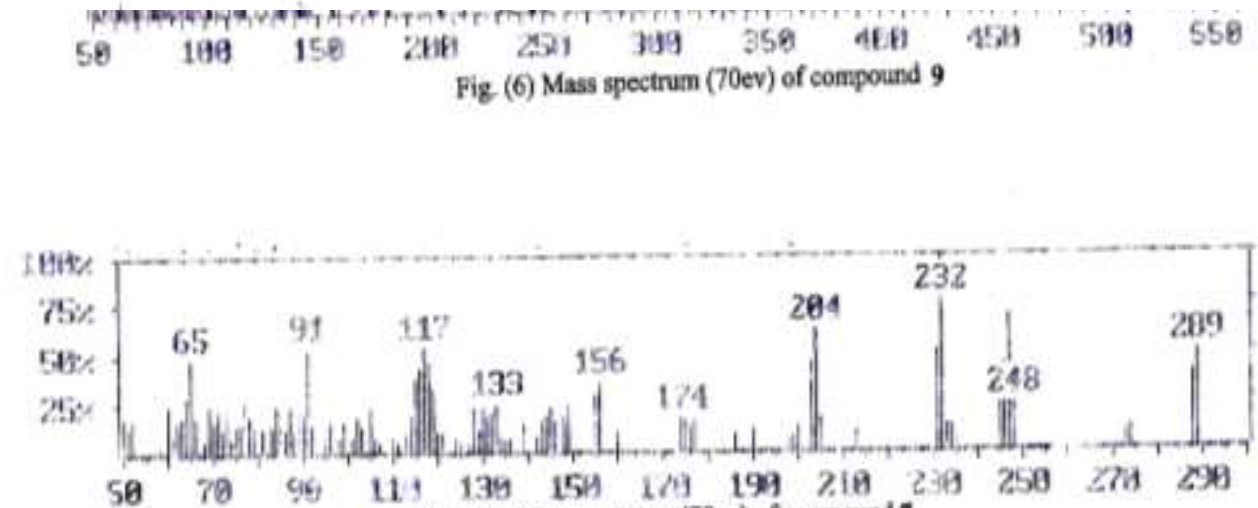
Fiz. (4) Mass spectrum (70ev) of compound 7
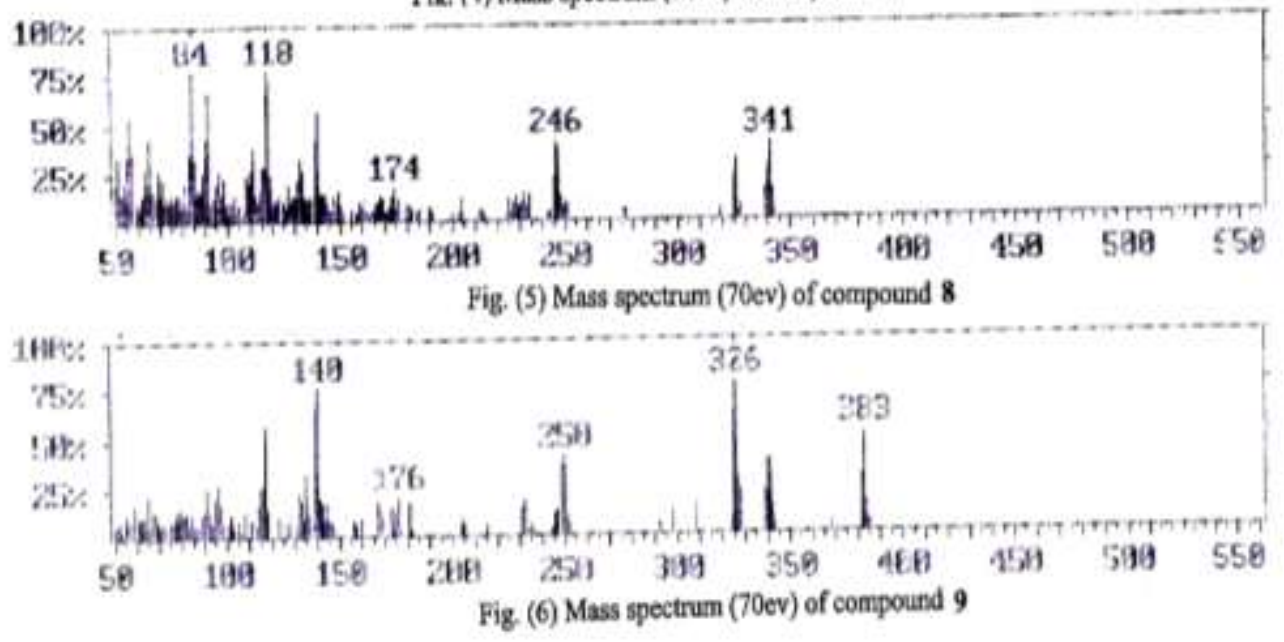


\section{Anti-microbial activity}

Using paper disk agar diffusion technique, (Rathore, et al., 2000; Rahman, et al., 2001) all the newly synthesized compounds were tested in vitro for antibacterial activity against the Staphylococcus aureus (RCMB 000108) and bacillus subtilis (RCMB 000109) (as gram positive bacteria) while Pesudomonas aeruginoca (RCMB 000103) and Escherichia coli( RCMB 000106) (as gram negative bacteria). Also these compounds were tested in vitro against some fungi as Aspergillus fumigatus (RCMB 002006), Geotrichum candidum (RCMB 05008), candida albicans (RCMB 005003), and Syncephalastrum racemosum (RCMB 005004) to know their antifungal activity. The compounds were tested for bacteria at $5 \mathrm{mg} / \mathrm{ml}$, but for fungi at $10 \mathrm{mg} / \mathrm{ml}$ concentration and the activity was determined by measuring the zone of inhibition.The screening results given in table $\mathbf{1}$ indicate that all the compounds exhibited antimicrobial activities .

Table 1 Antimicrobial activity of some synthesized compounds

\begin{tabular}{|c|c|c|c|c|c|c|c|c|c|c|}
\hline \multicolumn{2}{|c|}{ Microorganism } & 2 & 3 & 4 & 5 & 6 & 7 & 8 & 9 & 10 \\
\hline \multirow{2}{*}{$\begin{array}{l}\text { Gram } \\
\text { positive } \\
\text { bacteria }\end{array}$} & $\begin{array}{l}\text { Staphylococcus } \\
\text { aureus }\end{array}$ & ++ & ++ & - & ++ & +++ & ++ & ++ & +++ & +++ \\
\hline & Bacillus subtilis & +++ & ++ & - & +++ & +++ & ++ & +++ & +++ & +++ \\
\hline \multirow{2}{*}{$\begin{array}{l}\text { Gram } \\
\text { negative } \\
\text { bacteria }\end{array}$} & $\begin{array}{l}\text { Pseudomonas } \\
\text { aeruginoca }\end{array}$ & - & ++ & ++ & ++ & +++ & ++ & ++ & ++ & +++ \\
\hline & Escherichia coli & ++ & ++ & - & ++ & +++ & ++ & ++ & +++ & +++ \\
\hline \multirow{4}{*}{$\begin{array}{l}\text { Antifungal } \\
\text { activity }\end{array}$} & $\begin{array}{l}\text { Aspergillus } \\
\text { fumigatus }\end{array}$ & ++ & ++ & ++ & ++ & +++ & ++ & ++ & ++ & +++ \\
\hline & $\begin{array}{l}\text { Geotrichum } \\
\text { candidum }\end{array}$ & ++ & ++ & ++ & ++ & +++ & ++ & ++ & ++ & +++ \\
\hline & $\begin{array}{l}\text { Candida } \\
\text { albicans }\end{array}$ & ++ & ++ & ++ & ++ & ++ & ++ & ++ & ++ & ++ \\
\hline & $\begin{array}{l}\text { Syncephalastrum } \\
\text { racemousum }\end{array}$ & - & ++ & ++ & ++ & ++ & ++ & ++ & ++ & +++ \\
\hline
\end{tabular}

Note ( - ) No antimicrobial activity, ( + ) Mild activity, ( + + ) Moderate activity, $(+++)$ Marked activity.

\section{Minimum inhibition concentration}

Using agar plate method, (Damyanova, et al., 2000) the minimum inhibition concentration ( MIC ) of compounds 3, 5, 9 and $\mathbf{1 0}$ were determined against antimicrobial activity such as gram positive bacteria , gram negative bacteria and fungi. The minimum inhibition concentration ( MIC) results given in table 2 . The compound $\mathbf{1 0 g a v e}$ the lowest concentration compared to the other compounds that been tested against several microorganisms as shown in table $\mathbf{2}$. Compound $\mathbf{5}$ was the second drug giving lower( MIC) against the tested microorganisms except in case of Staphylococus aureus band Bacillus subtilis. However compound $\mathbf{3}$ demonstrated the higher concentration against the tested microorganisms. 
Table :2 Antimicrobial Activity as MIC ( $\mu \mathrm{g} / \mathrm{ml}$ )of tested samples against tested microorganisms.

\begin{tabular}{|l|l|l|l|l|l|}
\hline Microorganism & 3 & 5 & 9 & 10 \\
\hline $\begin{array}{l}\text { Gram positive } \\
\text { bacteria }\end{array}$ & $\begin{array}{l}\text { Staphylococcus } \\
\text { aureus }\end{array}$ & 62.5 & 15.6 & 7.8 & 1.9 \\
\hline & Bacillus subtilis & 13.2 & 7.8 & 3.9 & 0.95 \\
\hline $\begin{array}{l}\text { Gram negative } \\
\text { bacteria }\end{array}$ & $\begin{array}{l}\text { Pseudomon } \\
\text { asaeruginoca }\end{array}$ & 125 & 7.8 & 62.5 & 3.9 \\
\hline & Escherichia coli & 62.5 & 3.9 & 15.6 & 0.95 \\
\hline $\begin{array}{l}\text { Antifungal } \\
\text { activity }\end{array}$ & \begin{tabular}{l} 
Aspergillusfumigatus \\
\hline
\end{tabular} & 62.5 & 15.6 & 31.2 & 1.9 \\
\hline & $\begin{array}{l}\text { Geotrichum } \\
\text { candidum }\end{array}$ & 62.5 & 15.6 & 62.5 & 7.8 \\
\hline Candida albicans & 125 & 62.5 & 125 & 31.2 \\
\hline & $\begin{array}{l}\text { Syncephalastrum } \\
\text { racemousum }\end{array}$ & 500 & 125 & 250 & 31.2 \\
\hline
\end{tabular}

\section{CONCLUSION}

The researches study the successful synthesis and antimicrobial activity of some new thiohydantoin, thiazole derivatives .The investigation of antifungal and antibacterial activity data revealed that all the tested compounds showed moderate to good inhibition in DMSO. All the compounds exhibited antimicrobial activities. Compounds 6 and 10 showed higher activity against bacteria and fungi. Also, Compounds 3, 5, 7and 8 showed moderate activity against bacteria and fungi. Compound 9 showed higher activity against bacteria and moderate activity against fungi. The good activity is attributed to the presence of pharmacologically active $\mathrm{COOH}, \mathrm{COCH} 3, \mathrm{CH} 3$, thiazole , thiophene and thiohydantoin ring.

It is worth mentioning that the attachment of carboxylic group to thiohydantoin ring produce strong antimicrobial activity. Also the presence of acetyl group with thiophene ring in compound 9 showed higher activity against bacteria. As we consider all results obtained from antifungal and antibacterial tests together we can say that entire compounds tested are more active towards fungi and some bacteria. These preliminary results of biological activity of the tested compounds could offer an encouraging framework in this field that may lead to the discovery of novel antimicrobial agent

\section{Expermintal}

Melting points were taken in open capillaries with a Thomas uni-melt apparatus un corrected. NMR spectra were recorded on a general electric QE 300 instrument and chemical shifts are given with respect to TMS. IR spectra were recorded on a perkine-Elmer 1420 spectrometer and a Biorad FTS7 (KBR). Mass spectra were obtained on a Jeol JMS D-300 spectrometer operating at $70 \mathrm{ev}$. Microanalysis were conducted using an elemental analyzer 1106.

\section{4-Methyl acetophenone thiosemicarbazone ( 2 ).}

A mixture of 4 methyl acetophenone ( 1 ) $(0.01 \mathrm{~mol})$ and thiosemicarbazide $(0.01$ mol) in ethanol $(50 \mathrm{ml})$ was heated under reflux for $4 \mathrm{hr}$. the solid formed after cooling saw filtered of ,washed with water, dried and purified by crystallization with benzene to give $\mathbf{1}$ as 
colorless crystals yield $75 \%$ m.p. $\mathbf{1 5 5}^{\circ} \mathrm{C}$. IR(KBr): 3350, 3130(NH2), $3220(\mathrm{NH}), 1625$ $(\mathrm{C}=\mathrm{N}), 1350(\mathrm{C}=\mathrm{S}) \mathrm{cm}^{-1} .{ }^{1} \mathrm{H}-\mathrm{NMR}$ (DMSO-d6): $\delta 2.32$ (s, 3H, CH3), 2.15(s,3H, CH3), 7.02 (s, 2H, NH2), 7.24-7.78 (m, 4H, H-phenyl ring), 10.12 (s, 1H, NH) ppm. MS(m/z, \%) ;194( 3.9), 193 (23.3), 148 (2.3), 147 (3.9), 116 (9.3), 106 (5.4 ), 104 (10.9), 92 (8.5), 91 (21.7) 90 (6.3), 88 (13.2), 77 (11.6), 75 (9.3), 65 (13.2), 62 (20.9), 61 (17.8), 60 (100), 59 (11.6), 58 (8.5), 51 (34.4), 50 (6.2). Cald.:C,57.97;H, 6.28;N, 20.28; S, 15.45.Found :C,58.02; H, 6.12; N,19.97; S, 15.27.

\section{5-(p-Tolyl)-2- [(p-tolylethylidene)hydrazino] thiazole( 3 ).}

\section{3-[(p-tolylethylidene )amino]-2-thiohydantoin ( 6 ).}

A mixture of 2 (0.01 mol), $\omega$-bromo methyl aryl ketone,(such as 4-methyl phenacyl bromide $)(0.01 \mathrm{~mol})$, and ethyl chloro acetate in ethanol $(50 \mathrm{ml})$ in presence of fused sodium acetate $(0.03 \mathrm{~mol})$ was heated under reflux for $6 \mathrm{hr}$, then cooled and poured into water. The solid formed was filtered off, washed with water, dried and purified by suitable solvent to give 3 and $\mathbf{6}$ respectively .

5-(p-Tolyl)-2- [(p-tolylethylidene $)$ hydrazino] thiazole ( 3 ), yield 65\%, (EtOH), m.p.120 ${ }^{\circ} \mathrm{C}$. IR (KBr): 3225(NH), 1625(C=N), 1609,1592(C=C) $\mathrm{cm}^{-1} .{ }^{1} \mathrm{H}-\mathrm{NMR}$ (DMSO-d6): $\delta 2.21$ $(\mathrm{s}, 3 \mathrm{H}, \mathrm{CH} 3), 2.29(\mathrm{~s}, 3 \mathrm{H}, \mathrm{CH} 3), 2.25(\mathrm{~s}, 3 \mathrm{H}, \mathrm{CH} 3), 6.99-7.82(\mathrm{~m}, 9 \mathrm{H}, \mathrm{Ar}-\mathrm{H}, \mathrm{H}-\mathrm{phenyl}$ and thiazole ring), 10.71(s,1H,NH) ppm. MS (m/z, \% ); $324(.37), 309(0.1), 308(0.25), 306$ (2.11) 293 (0.93), 288 (1.13), 279 (0.15),278 (0.25),230 (1.80),229 (0.11),203 (5.35),190 (2.06),188 (15.08),173 (9.98),161 (6.39),158 (9.54),149 (2.86),147 (65.6), $142 \quad$ (4.45),134 (10.01),131 (41.00), 120 (2.80), 119 (3.41), 116 (6.39), 105 (5.00), 102 (10.93), 90 (100),83 (0.18), 78 (1.31), 76 (10.59), $72(0.18), 69$ (1.06) , 64 (34.3), 62 (12.6) , 57 (1.85) , 52 (2.85) ,50 (10.43),. Calcd.: C, 71.02 ;H, 5.91; N, 13.08; S, 9.96.Found:C, 71.15; H, 5.80; $\mathrm{N}, 13.15 ; \mathrm{S}, 9.84$.

3-[(p-tolylethylidene)amino]-2-thiohydantoin ( 6 ), yield, $80 \%$ (benzene); m.p.184 ${ }^{\circ} \mathrm{C}$. IR $(\mathrm{KBr}): 3250(\mathrm{NH}), 1625(\mathrm{C}=\mathrm{N}), 1357(\mathrm{C}=\mathrm{S}) \mathrm{cm}^{-1} .{ }^{1} \mathrm{H}-\mathrm{NMR}$ (DMSO-d6): $\delta 2.26(\mathrm{~s}, 3 \mathrm{H}, \mathrm{CH} 3)$, 2.14(s,3H,CH3), 3.65(s,2H,NCH2CO), 7.15-7.71(m,4H,H-phenyl ring), $10.98(\mathrm{~s}, 1 \mathrm{H}, \mathrm{NH})$ ppm. MS (m/z, \% ), 248 [ (M+1) 18.2 ] , 246 [(M-1) 42.7], 245 (25.5), 233 (3.6), 232 (18.2), 204 (13.5), 202 (2.1) , 201 (6.8) , 200 (6.8), 173 (4.2), 159 (17.2), 158 (5.7), 157 (2.6), 148 (1.6), 147 (9.4), 133 (18.8), 132 (14.6), 131 (12.5), 130 (17.2), 119 (13.0), 118 (100), 117 (38.5) , $115(30.2), 114(6.8) 92$ (15.1), 91 (39.6), 90 (07.3), 89 (17.2), 88 (15.6) , 87 (32.8), 77 (7.8), 65 (41.1), 64 (13.5), 63 (26.00), 62 (8.9) , 60 (18.20), 59 (6.3), 51 (10.9), 50 (15.1) .Calcd. ; C, 58.29; H, 5.26; N, 17.00; S,15.45.Found :C, 57.99; H, 5.46; N, $17.09 ; \mathrm{S}, 15.25$.

\section{2-[(p-tolylethylidene)acetyl hydrazino]-thiazole( 4 ).}

A solution of $3(0.01 \mathrm{~mol})$ in acetic anhydride $(30 \mathrm{ml})$ was heated under reflux for $2 \mathrm{hr}$, then cooled and poured onto ice-water. The solid formed dried and purified by crystallization from petroleum ether (60-80) to give 4 as orange crystals ,yield $60 \%$, ), m.p.140 ${ }^{\circ}$ C. IR (KBr): $1710(\mathrm{C}=\mathrm{O}), 1630(\mathrm{C}=\mathrm{N}), 1610,1593(\mathrm{C}=\mathrm{C}) \mathrm{cm}^{-1} .{ }^{1} \mathrm{H}-\mathrm{NMR}$ (DMSO$\left.\mathrm{d}_{6}\right): \delta 2.25\left(\mathrm{~s}, 3 \mathrm{H}, \mathrm{CH}_{3}\right), 2.25\left(\mathrm{~s}, 3 \mathrm{H}, \mathrm{CH}_{3}\right), 2.11\left(\mathrm{~s}, 3 \mathrm{H}, \mathrm{CH}_{3}\right), 2.3\left(\mathrm{~s}, 3 \mathrm{H}, \mathrm{COCH}_{3}\right), \quad 6.89-7.85$ (m, $9 \mathrm{H}, \mathrm{Ar}-\mathrm{H}, \mathrm{H}-$ phenyl and thiazole ring), ppm.

MS( m/z, \%);364 [ (M+1) 5.5 ] ,362[(M-1) 11], 347 (3.7), 346 (3.7), 323 (21.1), 322 (26.6),321 (100), 320 (74.3), 319 (32.1),307 (10), 306 (21.1), 293 (8.3),292 (10.1), 289 (8.3), 288 (11.9),275 (7.8), 263 (8.8), 248 (11.9) ,247 (22.0), 233 (8.3), 231(7.3),230 (7.3), 217(5.5), 216 (10.1), 205 (27.5), 204 (27.5), 203 (32.1),202 (16.5), 190 (18.3), 188 (15.6), 187 (11.0), 176(22.0), 175 (62.4), 174 (27.5), 171 (11,0), 163 (7.3), 162 (7.3), 161 (10.1), 158 (8.30), 148 (41.2), 147 (17.3), 146 (20.6), 144 (6.4),143 (15.6), 140 (7.3), 134(11.9), 133 (28.4), 132 (61.5), 131 (27.5), 118 (33.9), 117(64.2), 115 (22), 91(77.1), 90 (30.3), 88 (24.8), 
77 (22.9), 63 (20.2), 60 (20.2), 59 (15.6), 51 (20.2) . Calcd. :C,69.42; H, 5.78; N,11.57; S,8.81.Found: C,68.99; H, 5.86; N,11.25; S, 8.97.

\section{4-methylacetophenone - 2,4-diacetyl thiosemicarbazone ( 5 ).}

A solution of $2(0.01 \mathrm{~mol})$ in acetic anhydride $(25 \mathrm{ml})$ was heated under reflux for $2 \mathrm{hr}$, then cooled and poured onto ice water.The resulting solid was filtered off ,washed with water, dried and purified by recrystallization from benzene to give $\mathbf{5}$ as white crystals ,yield 70\% , m.p.202 ${ }^{\circ} \mathrm{C}$. IR(KBr): $3220(\mathrm{NH}), 1710,1695(\mathrm{C}=\mathrm{O}), 1620(\mathrm{C}=\mathrm{N}), 1354(\mathrm{C}=\mathrm{S})$ $\mathrm{cm}^{-1} .{ }^{1} \mathrm{H}-\mathrm{NMR} \quad\left(\mathrm{DMSO}_{-} \mathrm{d}_{6}\right): \quad \delta \quad 2.15\left(\mathrm{~s}, 3 \mathrm{H}, \mathrm{CH}_{3}\right), \quad 2.08\left(\mathrm{~s}, 3 \mathrm{H}, \mathrm{CH}_{3}\right), \quad 2.22\left(\mathrm{~s}, 3 \mathrm{H}, \mathrm{COCH}_{3}\right)$, 2.08(s,3H, $\left.\mathrm{COCH}_{3}\right), \quad 7.21-7.80(\mathrm{~m}, 4 \mathrm{H}, \mathrm{H}$-phenyl ring) ppm. MS (m/z, \% ); 292 [ (M+1) 24.1], 290 [ (M-1) 27.6] , 234 (100) ,193 (17.2), 192 (48.3), 190 (72.4), 176 (27.6) , 175 (17.2) , 174 (3.4), 158 (7.8), 157 (27.6), 150 (24.1), 149 (41.4), 148 (58.6), 147 (82.8), 136 (20.7),135 (31.0), 133 (51.7), 132 (75.9), 119 (41.4) ,118 (96.6),117 (79.3), 116 (44.8), 115 (27.6), 114 (34.5), 112 (24.1), 106 (27.6), 105 (17.2), 103 (34.5), 96 (17.2), 92 (48.3), 91 (51.7), 90 (58.6), 89 (34.5), 82 (31.0), 82 (31.0), 79 (10.3), 78 (41.4), 75 (24.1), 69 (17.2), 68 (31.0), $65(65.5), 64(17.2), 61(17.2), 60(31.0), 59(72.4), 57(41.4)$, 56 (27.4), 55 (89.7), 54 (31.0) , 52 (31.0) ,52 (34.0),50 (51.0). Calc.: C,57.73; H, 5.84; N, 14.43; S, 10.99. Found: C,57.60; H, 5.84; N, 14.43; S, 10.88.

\section{1-Acetyl-3-[(p-tolylethylidene) amino]-2-thiohydantoin ( 7 ).}

A solution of $6(0.01 \mathrm{~mol})$ in acetic anhydride $(25 \mathrm{ml})$ was heated under reflux for 2 $\mathrm{hr}$, then cooled and poured onto ice water.The resulting solid was filtered off , washed with water, dried and purified by recrystallization from benzene to give 7 as yellow crystals ,yield 68\% , m.p.158 ${ }^{\circ} \mathrm{C}$. IR(KBr):1703 -1672(C=O), $1627(\mathrm{C}=\mathrm{N}), 1353(\mathrm{C}=\mathrm{S}) \mathrm{cm}^{-1} .{ }^{1} \mathrm{H}-\mathrm{NMR}$ $\left(\mathrm{DMSO}_{6}\right): \delta 2.10\left(\mathrm{~s}, 3 \mathrm{H}, \mathrm{COCH}_{3}\right), 2.25\left(\mathrm{~s}, 3 \mathrm{H}, \mathrm{CH}_{3}\right), 2.19\left(\mathrm{~s}, 3 \mathrm{H}, \mathrm{CH}_{3}\right), 3.65\left(\mathrm{~s}, 2 \mathrm{H}, \mathrm{NCH}_{2} \mathrm{CO}\right)$, $7.11-7.75(\mathrm{~m}, 4 \mathrm{H}, \mathrm{H}$-phenyl ring) ppm.

MS (m/z, \% ); 288 [ (M-1) 38.5 ] , 274 (11.0) , 273 (9.9), 248 (23.1) , 246 (44.0), 245 (28.6), 235 (6.6) , 234 (13.2) , 233 (12.1), 232 (100), 231 (50.5), 213 (9.9), 255 (16.5), 203 (45.10), 200 (13.20), 199 (7.7), 198 (5.50) , 190 (11.0), 186 (8.8) , 177 (15.4) , 176 (12.1), 175 (15.4), 174 (16.5), 160 (9.9), 156 (35.2) , 155 (28.8) ,149 (24.2), 148 (17.6), $146(15,4)$, 144 (17.6) , 143 (15.4), 142 (7.7) ,136 (7.7) , 135 (5.50), 134 (6.60),132 (19.8), 131 (17.6), 130 (22.0), 128 (22.2), 125 (5.50), 124 (7.70), 116 (17.6), 115 (38.5), 110 (42.9),106 (7.7), 105 (22.01), 103 (12.1), 91 (52.7), 90 (19.8), 89 (20.9) , 76 (14.3), 73 (20.9), 69 (24.2), 52 (16.5), 51 (13.2), 50 (18.7) . Calcd.: C , 85.13; H , 5.19; N, 14.53; S, 11.07.Found : C, 57.99; $\mathrm{H}, 5.32 ; \mathrm{N}, 14.21 ; \mathrm{S}, 11.19$.

\section{5-Thiophen-2-ylidene-3-[(p-tolylethylidene)amino]-2-thiohydantoin ( 8 ).}

A solution of $6(0.01 \mathrm{~mol})$, thiophen-2-carboxaldehyde $(0.01 \mathrm{~mol})$ and pipredine $(1$ $\mathrm{ml}$ ) was fused on a hot plate at $\mathbf{1 0 0 - 1 1 0}^{\circ} \mathbf{C}$ for $2 \mathrm{hr}$. The reaction mixture was cooled and a acidified with dilute hydrochloric acid $(2 \mathrm{~N})$.The crude product was filtered off, washed with water, dried and purified by crystallization from ethanol to give $\mathbf{8}$ as pale yellow crystals yield $80 \%$, , m.p.100 ${ }^{\circ} \mathrm{C}$. IR(KBr): 3262(NH), 1695(C=O),1630(C=N), 1351(C=S), $1605,1585(\mathrm{C}=\mathrm{C}) \mathrm{cm}^{-1}$. ${ }^{1} \mathrm{H}-\mathrm{NMR}\left(\mathrm{DMSO}_{-} \mathrm{d}_{6}\right): \delta 2.23\left(\mathrm{~s}, 3 \mathrm{H}, \mathrm{CH}_{3}\right), 2.29\left(\mathrm{~s}, 3 \mathrm{H}_{1} \mathrm{CH}_{3}\right), 6.85-$ 7.98(m,8H,H-phenyl ,H-thiophene ring and olefinic proton), $10.53(\mathrm{~s}, 1 \mathrm{H}, \mathrm{NH}) \mathrm{ppm} . \mathrm{MS}(\mathrm{m} / \mathrm{z}$, $\%$ ): $342[(\mathrm{M}+1) 15.2$ ] , $340[(\mathrm{M}-1) 27.2], 339$ (15.9) ,328 (7.30), $326(30.5), 325$ (23.2), 251 (8.5), 250 (9.80), 248 (12.80), 247 (37.8), 246 (39.6), 145 (22.6),234 ( 12.4), 233 (6.7), 232 (13.4), 231 (9.1), 230 (7.30, 225 (11.00), 212 (505), 204 (11.00), 191 (4.3), 185 (6.1), 184 (4.30), 190 (6.70) , 182 (6.10), 180 (7.90), 176 (11.0), 174 (16.5), 173 (12.8), 171 (4.30), 170 (4.30), 169 (11.6), 168 (12.8), 165 (4.30), 164 (5.50), 162 (4.90),159 (9.10), 158 (4.90), 155 (6.10), 149 (15.9), 147 (12.8), 143 (14.0), 142 (14.0), 141 (13.4), 140 (56.7), 139 (40.9), 133 (26.8), 132 (32.3), 130 (12.8), 129 (12.2), 127 (18.9), 119 (23), 112 (13.40), 
111 (37.4),110 (26.2), 109 (19.50), 98 (22.6),96 (26.80), 95 (18.90), 94 (12.80), 92 (9.10), 91 (65.20), 90 (42.70) ,85(31.7), 84 (100.00), 83 (36.00), 79 (7.90), 78 (13.40), 77 (13.40), 76 (11.00), 75 (13.40), 73 (12.80),69 (25.6), 66 (16.5), 64 (2.07),65 (44.5) , 64 (2.7), 63 (27.40), 60 (11.6), 59 (10.40), 55 (13.4), 52 (14.6), 51 (33.5 ).Calc.: C, 59.82; H, 4.39; N, $12.31 ; \mathrm{S}, 18.76$.

\section{1-Acetyl-3- [(p-tolylethylidene)amino]-5-thiophen-2-ylidene-2-thiohydantoin (9).}

A solution of $\mathbf{8}(0.01 \mathrm{~mol})$ in acetic anhydride $(25 \mathrm{ml})$ was heated under reflux for 2 $\mathrm{hr}$, then cooled and poured onto ice water.The resulting solid was filtered off ,washed with water, dried and purified by recrystallization from benzene to give $\mathbf{9}$ as yellow crystals ,yield $80 \%$, m.p.80 ${ }^{\circ} \mathrm{C}$. IR(KBr): 1710-1703(br-C=O), $1629 \quad(\mathrm{C}=\mathrm{N}), \quad 1606,1586$ $(\mathrm{C}=\mathrm{C}), 1352(\mathrm{C}=\mathrm{S}) \mathrm{cm}^{-1},{ }^{1} \mathrm{H}-\mathrm{NMR}\left(\mathrm{DMSO}-\mathrm{d}_{6}\right): \delta 2.01\left(\mathrm{~s}, 3 \mathrm{H}, \mathrm{COCH}_{3}\right), 2.27\left(\mathrm{~s}, 3 \mathrm{H}, \mathrm{CH}_{3}\right), 2.18$ $\left(\mathrm{s}, 3 \mathrm{H}, \mathrm{CH}_{3}\right), 6.89-7.97(\mathrm{~m}, 8 \mathrm{H}, \mathrm{H}$-phenyl,H-thiophen ring and olefinic protone $) \mathrm{ppm}$. MS $(\mathrm{m} / \mathrm{z}, \%): 358[(\mathrm{M}+2) 6.3], 384[(\mathrm{M}+1)$ 15.2] $, 382[(\mathrm{M}-1), 29], 36$ (5.4), 343 (6.3), 342 (13.4), 341 (37.5), 340 (37.5),339 (22.3), 328 (22.3), 327 (30.4), 326 (100), 325 (61.6), 308 (13.5), 298 (11.60), 252 (5.40), 251 (9.9) , 250 (40.2), 235 (5.4), 232 (17.9), 231 (13.4), 215 (5.4), 205 (5.5), 204 (11.6), 182 (3.6), 181 (16.1), 176 (19.6), 175 (10.7), 173 (13.4), 168 (14.4), 160 (8.0), 156 (8.9),148 (0.99), 146 (7.1), 145 (8.90), 144 (17.0), $142(16.1), 141$ (19.6), 140 (75.0), 139 (64.3), 138 (7.1), 135 (32.1), 134 (8.9), 133 (17.9), 132 (22.3), 131 (5.4), 127 (7.1), 123 (9.80), 118 (13.4), 117 (56.3) , 116 (25.9), 115 (25.9), 114(15.2),110 (12.5),106 (2.7), $102(11.60)$,

101 (7.10), 99 (3.60) , 97 (16.10) , 96 (25.4), 95 (19.0), 92 (12.50), 91 (25.0), 90 (14.30), 88 (7.10), 84 (11.6), 83 (5.4), 82 (11.3), 81 (11.6) 80 (11.6), 79 (13.4), 73 (6.50), 69 (8.90), 68 (12.50),64 (5.40, 63 (5.90), 60 (00.90), 56 (5.40), 55 (10.70), 53 (3.60), 52 (8.0), 51 (6.3), 50 (6.3) . Calcd. :C, 59.53; H, 4.43; N, 10.96; S, 16.71.Found : C, 58.99; H4.65; N, $10.84 ; \mathrm{S}, 16.65$.

\section{3-[(p-tolylethylidene)amino]-2-thiohydantoin-5-yl acetic acid ( 10 ).}

A mixture of $6(0.01 \mathrm{~mol})$, chloroacetic acid $(0.01 \mathrm{~mol})$ and sodium metal $(0.5 \mathrm{gm})$ in xyline $(50 \mathrm{ml})$ was heated under reflux for $4 \mathrm{hr}$. Then filtered upon hot and the filtrate concentrated the solid formed was filtered off ,dried and purified by crystallization from ethanol to give $\mathbf{1 0}$ as yellow crystals, yield $65 \%$, m.p.220 ${ }^{\circ} \mathrm{C}$. IR(KBr): $3230(\mathrm{NH})$, 1720,1703(C=O), 1625(C=N), 1355(C=S), $3308-2873(\mathrm{br}-\mathrm{OH}) \mathrm{cm}^{-1} .{ }^{1} \mathrm{H}-\mathrm{NMR}$ (DMSO$\left.\mathrm{d}_{6}\right): \delta 2.25\left(\mathrm{~s}, 3 \mathrm{H}, \mathrm{CH}_{3}\right), \quad 2.16\left(\mathrm{~s}, 3 \mathrm{H}, \mathrm{CH}_{3}\right), \quad 3.65\left(\mathrm{~s}, 2 \mathrm{H}, \mathrm{NCH}_{2} \mathrm{CO}\right), \quad 7.21-7.95(\mathrm{~m}, 7 \mathrm{H}, \mathrm{H}-$ pyenyl ring, oleifinic protons), 10.50(s,1H,NH), 11.52(s,1H,OH) ppm . MS (m/z, \% ) : $307[(\mathrm{M}+2)$ 0.33], 293 (0.16), 264 (0.81), 262 (0.15), 261 (0.15), 249 (3.19), 246 (2.09), 232 (1.24), 230 (3.49), 205 (2.85), 190 (3.45), 186 (2.14), 183(1.63), 177 (1.12), 176 (1.13)174 (5.66), 161 (5.03), 159 (2.38), 156 (2.86), 14 (31.74), 135 (1.89), 134 (16.4), 118 (100), 115 (34.16), 106 (6.7), 104 (43.7), 93 (7.37), 91 (48.80), 89 (11.76), 86 (5.14), 82 (1.63), 77 (16.79), 65 (15.83), 62 (11.75), 58(11.8),55 (5.86), 52 (5.86), 50 (14.99) . Calcd. :C, 55.08; H, 4.91; N, 13.77; S, 10.49.Found : C, 55.17; H, 4.85; N, 13.05; S 10.6 


\section{REFERANCES}

Bêhai, I.; (2003): Tetrahedron Lett., 44, 7475.

Brouilette, W.L.; Jestkov, V.P.; Brown, M.L.; Akhtar, M.S.; Deloney, T.M. and Brown, G.M.; (1994): J. Med.chem., 37, 3289.

Damyanova,S.; Gomez L.M.; Banares, M.A. and Fierro,J.L.G.; (2000): Chem.Mater. 12 , 501777.

El-Deen, I.M. and Ibrahim, H.K.; (2004): Chemical Paper, 58, 200.

Hasanen, J.A.; (2007): Egypt. J. Chem., 50 (2), 203.

Kiec.Kononowicz, K. and Szyman'ska, E.; (2002): I I Farmaco, 57, 909.

Mohamed, S.N.; Unis, N. and Abd El-Hady, H.; ( 2006a): Egypt. J.chem., 49 (2 ), 209

Mohamed, S.N.; Unis, N. and Abd El-Hady, H.; (2006b): Indian J.Chem., 45.

Rahman A.;Choudhary, M.I. and Thomsen W. J.; (2001): Bioassay Techniques for Drug Development . Harwood Academic Publishers, the Netherlands pp.16..

Rathore H.s.;Mittal S. and Kumar S.; (2000): Pestic . Res .J.12103.

Shivakama, H.B.; Shivanada,M.K. and Akberali, P.M.; (1998 ): J. Indian Chem.Soc., 75, 532.

Wilson, L.J. and Portlock, D.E.; (1998): Tetrahedron Lett., 39, 5135.

تحضير وتحقيق طيف الكتلة والنشاط البيولوجى لبعض مشتقات الثيوهيانتوين والثيازول الجديدة

$$
\begin{aligned}
& \text { هبة عبدالهادى محمد عبدالهادى } \\
& \text { قسم الكيمياء - كلية العلوم (بنات)- جامعة الأزهر ـ القاهرة }
\end{aligned}
$$

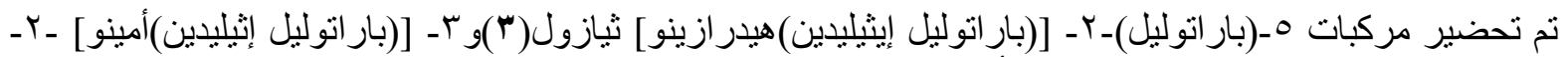

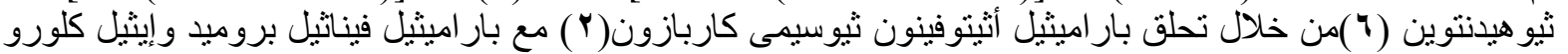

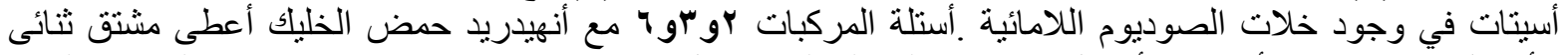

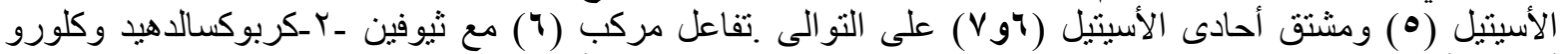

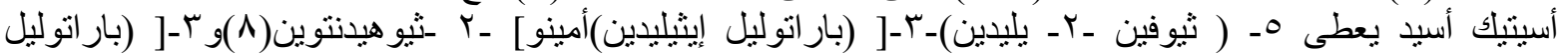

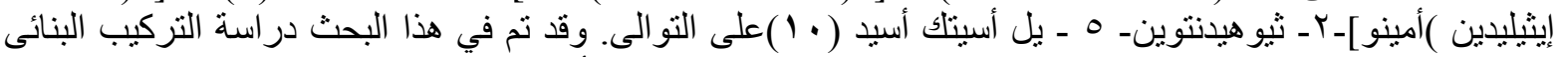

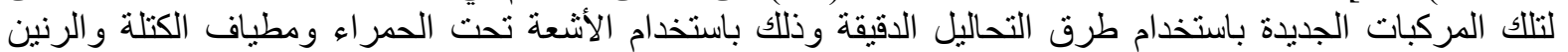

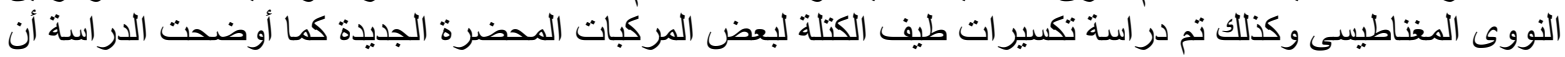

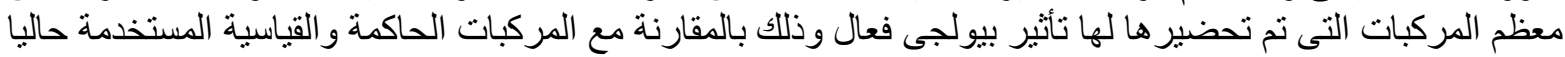
كمثبطات لنمو الميكروبات مما يوضح إمكانية الإستفادة من هذه المركبات في المجال التطبيقى كمثبطات لنمو البكات البكتريا و الفطريات. 